\title{
Absorbing interfaces in structural-acoustic coupled problems
}

\author{
Walid Larbi — Jean-François Deü — Roger Ohayon \\ Structural Mechanics and Coupled Systems Laboratory \\ Conservatoire National des Arts et Métiers \\ Chaire de Mécanique - Case courrier 353 \\ 2 rue Conté, F-75003 Paris \\ \{walid.larbi, jean-francois.deu, roger.ohayon\}@cnam.fr
}

\begin{abstract}
This work concerns finite element formulations of structural-acoustic interior problems with dissipative interfaces. The main purpose is to establish the link between wall acoustic impedance models and poroelastic appraoches based on the Biot theory. The proposed method consists in determining the acoustic impedance parameters starting from intrinsic characteristics of the porous medium. This impedance is then introduced into the vibroacoustic finite element formulation to take into account the dissipative aspect of the fluid-structure interface.

RÉSUMÉ. Ce travail porte sur la modélisation éléments finis de problèmes de vibroacoustique interne avec interfaces dissipatives. L'objectif est de faire le lien entre une modélisation du matériau absorbant par impédance de paroi et une modélisation de type poroélastique s'appuyant sur la théorie de Biot. L'approche proposée consiste à déterminer les paramètres de l'impédance de paroi à partir de caractéristiques intrinsèques du milieu poreux. Cette impédance est ensuite introduite dans la formulation éléments finis du problème vibroacoustique pour tenir compte de l'aspect dissipatif à l'interface fluide-structure.

KEYWORDS: elastoacoustic, noise reduction, porous medium, wall acoustic impedance, finite elements.

MOTS-CLÉS : élastoacoustique, réduction du bruit, milieu poreux, impédance acoustique de paroi, éléments finis.
\end{abstract}

DOI:10.3166/REMN.17.677-688 @ 2008 Lavoisier, Paris 


\section{Introduction}

In recent years, considerable research has been devoted to the development and testing of noise reduction techniques by passive damping treatments. Many approaches have been proposed in the literature, in particular to model the absorbing material. These approaches are often based on poroelastic material modeling (Allard, 1993; Atalla et al., 1998; Göransson, 1998; Davidsson, 2004). In this work, we focus on the formulation of structural-acoustic problems with interface damping using a wall impedance approach. In this context, let us mention the paper of Kehr-Candille and Ohayon (Kehr-Candille et al., 1992), where a frequency-dependent impedance is introduced to describe the absorbing material at the fluid-structure interface, and where a substructuring method is used to solve the dissipative structuralacoustic system. In that work, the fluid is described by a scalar unknown field (pressure or fluid displacement potential) and the problem is numerically solved in frequency domain by the finite element method. Using the same kind of approach, Bermúdez and Rodríguez present in (Bermúdez et al., 1999) a finite element method to compute the dynamic response of an elastoacoustic system with dissipative interface subject to external harmonic excitations. In their paper, a displacement formulation is used for both media, requiring a particular attention to the discretization of the admissible class of irrotational motions of the fluid. More recently, an original formulation for interior structural-acoustic dissipative problems, based on the introduction of the normal fluid displacement field at the fluid-structure interface, has been proposed by the authors (Deï et al., 2006; Larbi et al., 2006). In the present paper, the main purpose is to establish the link between dissipative models by wall acoustic impedance and by poroelastic approach based on the Biot-Allard theory (Allard, 1993) (cf. Figure1).

(a)

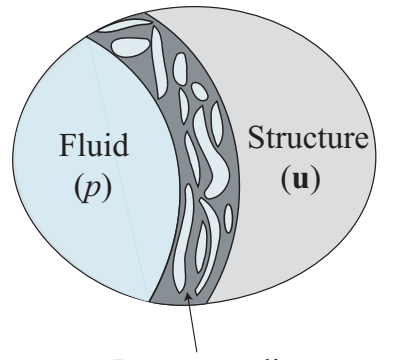

Porous medium $\left(\mathbf{u}^{s}, p^{f}\right)$ (b)

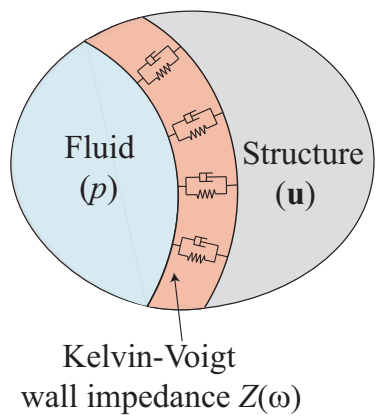

Figure 1. Structural-acoustic problem with absorbing interface modeled by: a) porous medium, b) acoustic wall impedance

The dissipative structural-acoustic coupled problem consists of an elastic structure covered by a thin layer of absorbing material and filled with an inviscid, compress- 
ible and barotropic fluid, gravity effects being neglected. In order to take the effect of a thin layer of absorbing material at the fluid-structure interface into account, a wall impedance approach is used. The question is here how to model and identify this impedance. A first strategy consists in using experimental measurements in a particular frequency range (Pierce, 1989). Another choice, which is developed in this paper, is to use refined poroelactic approaches. In this context, we present in the first part of this paper the finite element modeling of poroelastic absorbing material based on Biot-Allard theory. The associated acoustic wall impedance can then be predicted from this numerical approach or by an analytical expression in the case of laterally infinite poroelastic materials excited by unit amplitude normal incidence plane wave. Next, we present the finite element formulations of structural-acoustic problem with absorbing interface considering (i) general acoustic impedance model and (ii) approximate Kelvin-Voigt model. In this last case, the associated matrix system can be solved in frequency and time domains using direct time integration methods and/or modal reduction approaches.

\section{Poroelastic modeling of absorbing materials based on Biot-Allard theory}

In the literature, several 3D finite element models based on Biot-Allard theory are developed for the forced response of a poroelastic material (Göransson, 1998; Atalla et al., 1998; Davidsson et al., 2006). We recall in this section the pressure/displacement $\left(\mathbf{u}^{s}, p^{f}\right)$ finite element formulation developed by Atalla (Atalla et al., 1998). This numerical approach will be used to model and identify the normal incidence surface impedance $Z(\omega)$.

\subsection{Finite element formulation}

We consider a single isotropic porous material described by the Biot-Allard approach (Allard, 1993). This model is defined by (i) five geometrical parameters (the porosity $\phi$, the flow resistivity $\sigma$, the tortuosity $\alpha_{\infty}$, the viscous characteristic length $\Lambda$, and the thermal characteristic length $\Lambda^{\prime}$ ), (ii) the mechanical characteristics of the skeleton, and (iii) the saturating fluid properties. A detailed description of this model can be found for example in (Atalla et al., 1998).

The Biot-Allard poroelasticity equations are written here in terms of the solid phase displacement $\mathbf{u}^{s}$ and the interstitial fluid pressure $p^{f}$. It is important to note that the small movement of the fluid is not irrotational and the dissipation effects are expressed by complex quantities which depend on the angular frequency $\omega$ (cf. signs " " in the following equations). 
For harmonic motion, the local equations of the poroelastic medium are given in terms of $\left(\mathbf{u}^{s}, p^{f}\right)$ by

$$
\begin{aligned}
& \operatorname{div} \boldsymbol{\sigma}^{s}\left(\mathbf{u}^{s}\right)+\omega^{2} \tilde{\rho} \mathbf{u}^{s}+\tilde{\gamma} \nabla p^{f}=\mathbf{0} \\
& \Delta p^{f}+\omega^{2} \frac{\tilde{\rho}_{22}}{\tilde{R}} p^{f}-\omega^{2} \frac{\tilde{\rho}_{22}}{\phi^{2}} \tilde{\gamma} \operatorname{div} \mathbf{u}^{s}=0
\end{aligned}
$$

where $\sigma^{s}$ is the in vacuo stress tensor of the poroelastic aggregate. Moreover, we have $\tilde{\rho}=\tilde{\rho}_{11}-\tilde{\rho}_{12}^{2} / \tilde{\rho}_{22}, \tilde{\gamma}=\phi\left(\tilde{\rho}_{12} / \tilde{\rho}_{22}-\tilde{Q} / \tilde{R}\right), \tilde{Q}=(1-\phi) \tilde{K}_{e}, \tilde{R}=\phi \tilde{K}_{e}$ where $\tilde{K}_{e}$ is the bulk modulus of the fluid phase; $\tilde{\rho}_{11}$ is the corrected mass density for the solid phase; $\tilde{\rho}_{12}$ is the inertial coupling factor; and $\tilde{\rho}_{22}$ is the corrected mass density of the fluid phase. All these parameters are defined in terms of the previously mentioned geometrical and mechanical properties of the porous medium.

From Equations [1] and [2], the variational formulation can be written in the following form

$$
\begin{gathered}
\int_{\Omega_{p}} \boldsymbol{\sigma}^{s}: \boldsymbol{\varepsilon}^{s}\left(\delta \mathbf{u}^{s}\right) \mathrm{d} v-\omega^{2} \int_{\Omega_{p}} \tilde{\rho} \mathbf{u}^{s} \cdot \delta \mathbf{u}^{s} \mathrm{~d} v \\
-\int_{\Omega_{p}} \tilde{\gamma} \nabla p^{f} \cdot \delta \mathbf{u}^{s} \mathrm{~d} v-\int_{\partial \Omega_{p}}\left(\boldsymbol{\sigma}^{s} \mathbf{n}\right) \cdot \delta \mathbf{u}^{s} \mathrm{~d} s=0 \quad \forall \delta \mathbf{u}^{s} \\
\int_{\Omega_{p}} \frac{\phi^{2}}{\omega^{2} \tilde{\rho}_{22}} \nabla p^{f} \cdot \nabla \delta p^{f} \mathrm{~d} v-\int_{\Omega_{p}} \frac{\phi^{2}}{\tilde{R}} p^{f} \delta p^{f} \mathrm{~d} v-\int_{\Omega_{p}} \tilde{\gamma} \mathbf{u}^{s} \cdot \nabla \delta p^{f} \mathrm{~d} v \\
+\int_{\partial \Omega_{p}}\left(\tilde{\gamma} \mathbf{u}^{s} \cdot \mathbf{n}-\frac{\phi^{2}}{\omega^{2} \tilde{\rho}_{22}} \frac{\partial p^{f}}{\partial n}\right) \delta p^{f} \mathrm{~d} s=0 \quad \forall \delta p^{f}
\end{gathered}
$$

where $\Omega_{p}$ and $\partial \Omega_{p}$ refer to the poroelastic domain and its boundary surface, $\mathbf{n}$ is the external normal vector of the boundary $\partial \Omega_{p}, \varepsilon^{s}$ is the strain tensor of the solid phase.

The discretization of the previous variational formulation leads to the following matrix equation

$$
\left(\begin{array}{cc}
\mathbf{K} & -\tilde{\mathbf{C}} \\
\mathbf{0} & \tilde{\mathbf{H}}
\end{array}\right)\left(\begin{array}{l}
\mathbf{U}^{s} \\
\mathbf{P}^{f}
\end{array}\right)-\omega^{2}\left(\begin{array}{cc}
\tilde{\mathbf{M}} & \mathbf{0} \\
\tilde{\mathbf{C}}^{T} & \tilde{\mathbf{G}}
\end{array}\right)\left(\begin{array}{l}
\mathbf{U}^{s} \\
\mathbf{P}^{f}
\end{array}\right)=\left(\begin{array}{l}
\mathbf{F}^{s} \\
\mathbf{F}^{f}
\end{array}\right)
$$


where $\mathbf{U}^{s}$ and $\mathbf{P}^{f}$ are the vectors of nodal values of $\mathbf{u}^{s}$ and $p^{f}$ respectively, and where the submatrices of Equation [5] are given by

$$
\begin{gathered}
\int_{\Omega_{p}} \boldsymbol{\sigma}^{s}\left(\mathbf{u}^{s}\right): \varepsilon^{s}\left(\delta \mathbf{u}^{s}\right) \mathrm{d} v \Rightarrow \delta \mathbf{U}^{s T} \mathbf{K} \mathbf{U}^{s} ; \int_{\Omega_{p}} \tilde{\rho} \mathbf{u}^{s} \cdot \delta \mathbf{u}^{s} \mathrm{~d} v \Rightarrow \delta \mathbf{U}^{s T} \tilde{\mathbf{M}} \mathbf{U}^{s} \\
\int_{\Omega_{p}} \tilde{\gamma} \nabla p^{f} \cdot \delta \mathbf{u}^{s} \mathrm{~d} v \Rightarrow \delta \mathbf{U}^{s T} \tilde{\mathbf{C}} \mathbf{P}^{f} ; \int_{\Omega_{p}} \tilde{\gamma} \mathbf{u}^{s} \cdot \nabla \delta p^{f} \mathrm{~d} v \Rightarrow \delta \mathbf{P}^{f^{T}} \tilde{\mathbf{C}}^{T} \mathbf{U}^{s} \\
\int_{\Omega_{p}} \frac{\phi^{2}}{\tilde{\rho}_{22}} \nabla p^{f} \cdot \nabla \delta p^{f} \mathrm{~d} v \Rightarrow \delta \mathbf{P}^{f^{T}} \tilde{\mathbf{H}} \mathbf{P}^{f} ; \int_{\Omega_{p}} \frac{\phi^{2}}{\tilde{R}} p^{f} \delta p^{f} \mathrm{~d} v \Rightarrow \delta \mathbf{P}^{f^{T}} \tilde{\mathbf{G}} \mathbf{P}^{f}
\end{gathered}
$$

In structural-acoustic problem with absorbing material, this approach leads to a coupling between porous material degrees of freedom and the surrounding fluid and structure degrees of freedom. Therefore, the whole problem is very large and thus computationally time consuming. One of the best ways to avoid this problem is to use a reduced or impedance models as described in the next sections.

\subsection{Computation of normal incidence surface impedance}

The surface impedance of laterally infinite poroelastic material predicted by the presented $\left(\mathbf{u}^{s}, p^{f}\right)$ model is investigated in this section. The configuration under study is depicted in Figure 2. The porous layer, of thickness $L=0.1 \mathrm{~m}$ and material properties given in Table 1 is bonded onto a rigid wall at $x=0$. A normal incidence plane wave of unit amplitude excites the absorbing material at $x=-L$.

Normal incidence

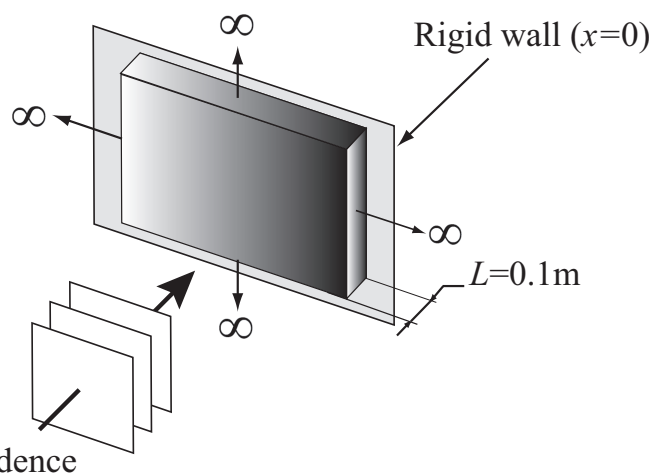

plane wave $(x=-L)$

Figure 2. Geometry of the normal acoustic impedance problem 
Table 1. Physical properties of the porous material

\begin{tabular}{ccccccccc}
\hline \hline$\phi$ & $\sigma\left(\mathrm{Ns} / \mathrm{m}^{4}\right)$ & $\alpha_{\infty}$ & $\Lambda(\mu \mathrm{m})$ & $\Lambda^{\prime}(\mu \mathrm{m})$ & $E_{s}(\mathrm{kPa})$ & $\nu_{s}$ & $\eta_{s}$ & $\rho_{s}\left(\mathrm{~kg} / \mathrm{m}^{3}\right)$ \\
\hline .94 & 40000 & 1.06 & 56 & 110 & 4400 & 0 & .1 & 130 \\
\hline \hline
\end{tabular}

The normal incidence surface impedance (Figure $3 \mathrm{a}$ ) is calculated using the displacement of fluid and solid phases, $\mathbf{u}^{s}$ and $\mathbf{u}^{f}$ respectively, at the input surface $(x=-L)$ for the unit acoustic pressure excitation by the following equation

$$
Z(\omega)=\frac{1}{i \omega\left[\phi \mathbf{u}^{f}(-L)+(1-\phi) \mathbf{u}^{s}(-L)\right]}
$$

where the displacement of fluid and solid phases are calculated using the previous $\left(\mathbf{u}^{s}, p^{f}\right)$ formulation.
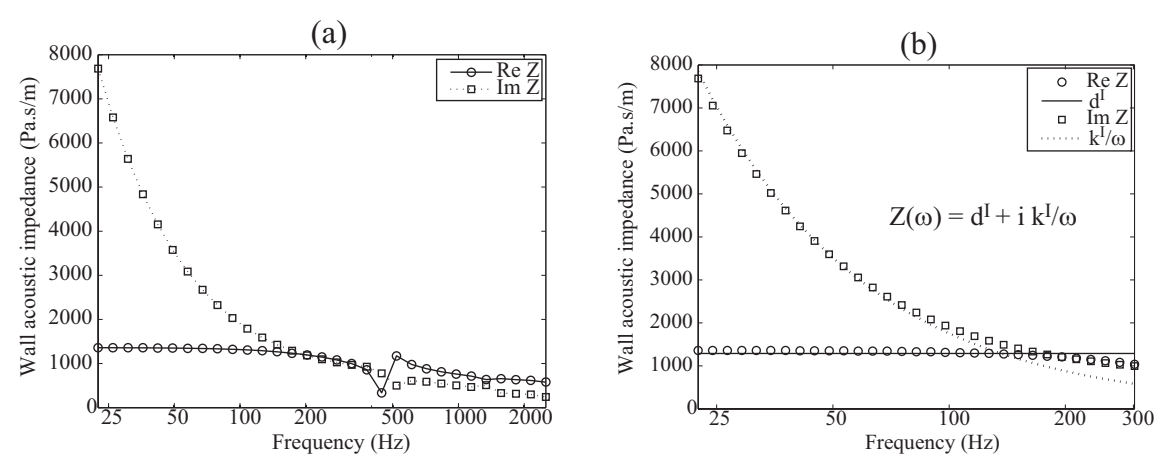

Figure 3. Real and imaginary parts of normal incidence surface impedance: (a) normal incidence surface impedance computed from Biot-Allard theory, $(b)$ identification of normal impedance parameters $k^{I}$ and $d^{I}$ in frequency range [0 - $\left.300 \mathrm{~Hz}\right]$

In frequency range $[0-300 \mathrm{~Hz}]$, the normal incidence surface impedance can be approached by a Kelvin-Voigt model, i.e. sum of a constant real part and an imaginary part inversely proportional to the frequency:

$$
Z(\omega)=d^{I}+i k^{I} / \omega
$$

The parameters $k^{I}$ and $d^{I}\left(k^{I} \approx 1.1 \times 10^{6} \mathrm{~Pa} / \mathrm{m}\right.$ and $\left.d^{I} \approx 1290 \mathrm{~Pa} . \mathrm{s} / \mathrm{m}\right)$, characterizing respectively the elastic and the viscous aspect of the absorbing layer, are deduced from the acoustic surface impedance by a least squares method (Figure $3 \mathrm{~b}$ ).

\section{Finite element formulations of structural-acoustic problems with absorbing interface}

This section concerns the finite element modeling of structural-acoustic problems with absorbing interfaces. The absorbing material is modeled by a frequency- 
dependent wall acoustic impedance. We present here a pressure/displacement formulation with (i) general acoustic impedance and (ii) Kelvin-Voigt impedance models.

\subsection{General acoustic impedance model}

We consider an elastic structure occupying the domain $\Omega_{S}$ at the equilibrium. The structure is clamped on a part $\Gamma_{u}$ and subjected to surface force density $\mathbf{F}^{d}$ on the complementary part of its external boundary $\Gamma_{t}$. The damping interface $\Sigma$ between the fluid and the structure corresponds to a third domain without thickness. This domain is modeled by a wall acoustic impedance $Z(\omega)$. The local equations of this structuralacoustic coupled problem with damped interface can be written in terms of structure displacement $\mathbf{u}$ and fluid pressure field $p$ by

$$
\begin{aligned}
\operatorname{div} \boldsymbol{\sigma}(\mathbf{u})+\rho_{S} \omega^{2} \mathbf{u} & =\mathbf{0} & & \text { in } \Omega_{S} \\
\boldsymbol{\sigma}(\mathbf{u}) \mathbf{n}_{S} & =\mathbf{F}^{d} & & \text { on } \Gamma_{t} \\
\boldsymbol{\sigma}(\mathbf{u}) \mathbf{n}_{S} & =p \mathbf{n} & & \text { on } \Sigma \\
\mathbf{u} & =0 & & \text { on } \Gamma_{u} \\
\Delta p+\frac{\omega^{2}}{c_{F}^{2}} p & =\mathbf{0} & & \text { in } \Omega_{F} \\
\nabla p \cdot \mathbf{n} & =\rho_{F} \omega^{2} \mathbf{u} \cdot \mathbf{n}+i \frac{\rho_{F} \omega}{Z(\omega)} p & & \text { on } \Sigma
\end{aligned}
$$

where $\mathbf{n}_{S}$ and $\mathbf{n}$ are the external unit normal to $\Omega_{S}$ and $\Omega_{F} ; \rho_{S}$ and $\rho_{F}$ are the structure and fluid mass densities; $c_{F}$ is the speed of sound in the fluid; and $\sigma$ is the structure stress tensor.

The variational formulation of the problem is obtained using the test-function method. For this purpose, we introduce the spaces $C_{u}$ and $C_{p}$ of sufficiently smooth functions associated with the field variables $\mathbf{u}$ and $p$ respectively.

Let $\delta \mathbf{u}$ be the test function associated to $\mathbf{u}$, belonging to the admissible space $C_{u}^{\star}=\left\{\delta \mathbf{u} \in C_{u} \mid \delta \mathbf{u}=\mathbf{0}\right.$ on $\left.\Gamma_{u}\right\}$. Multiplying Equation [8] by $\delta \mathbf{u} \in C_{u}^{\star}$, applying Green's formula, and finally taking Equations [9] and [10] into account, we have:

$$
\begin{aligned}
\int_{\Omega_{S}} \boldsymbol{\sigma}(\mathbf{u}): \varepsilon(\delta \mathbf{u}) \mathrm{d} v-\int_{\Sigma} p \mathbf{n} \cdot \delta \mathbf{u} \mathrm{d} s- & \omega^{2} \int_{\Omega_{S}} \rho_{S} \mathbf{u} \cdot \delta \mathbf{u} \mathrm{d} v \\
& =\int_{\Gamma_{t}} \mathbf{F}^{d} \cdot \delta \mathbf{u} \mathrm{d} s \quad \forall \delta \mathbf{u} \in C_{u}^{*}
\end{aligned}
$$


Similarly, let $\delta p$ be the test function, associated to $p$, belonging to the admissible space $C_{p}$. Multiplying Equation [12] by $\delta p \in C_{p}$, applying Green's formula, and finally taking Equation [13] into account, we obtain:

$$
\begin{aligned}
\frac{1}{\rho_{F}} \int_{\Omega_{F}} \nabla p \cdot \nabla \delta p \mathrm{~d} v-i \frac{\omega}{Z(\omega)} \int_{\Sigma} p \delta p \mathrm{~d} s-\omega^{2} \int_{\Sigma} \mathbf{u} \cdot \mathbf{n} \delta p \mathrm{~d} s & \\
& -\frac{\omega^{2}}{\rho_{F} c_{F}^{2}} \int_{\Omega_{F}} p \delta p \mathrm{~d} v=0 \quad \forall \delta p \in C_{p}
\end{aligned}
$$

Thus, the variational unsymmetric formulation of the elastoacoustic problem with interface damping consists, for given appropriate initial conditions, in finding $(\mathbf{u}, p) \in$ $\left(C_{u}^{\star}, C_{p}\right)$ such that, $\forall(\delta \mathbf{u}, \delta p) \in\left(C_{u}^{\star}, C_{p}\right)$ Equations [14] and [15] are satisfied.

After discretizing by the finite element method the bilinear forms in Equations [14 and [15], we obtain the following matrix system of the coupled problem:

$$
\left[\left(\begin{array}{cc}
\mathbf{K}_{u} & -\mathbf{C}_{u p} \\
\mathbf{0} & \mathbf{K}_{p}
\end{array}\right)-i \frac{\omega}{Z(\omega)}\left(\begin{array}{cc}
\mathbf{0} & \mathbf{0} \\
\mathbf{0} & \mathbf{D}_{p}
\end{array}\right)-\omega^{2}\left(\begin{array}{cc}
\mathbf{M}_{u} & \mathbf{0} \\
\mathbf{C}_{u p}^{T} & \mathbf{M}_{p}
\end{array}\right)\right]\left(\begin{array}{l}
\mathbf{U} \\
\mathbf{P}
\end{array}\right)=\left(\begin{array}{l}
\mathbf{F} \\
\mathbf{0}
\end{array}\right)
$$

where $\mathbf{U}$ and $\mathbf{P}$ are the vectors of nodal values of $\mathbf{u}$ and $p$ respectively; $\mathbf{F}$ is the vector of external forces defined by $\int_{\Gamma_{t}} \mathbf{F}^{d} \cdot \delta \mathbf{u} \mathrm{d} s \Rightarrow \delta \mathbf{U}^{T} \mathbf{F}$; and the real and frequencyindependent submatrices of Equation [16] are given by:

$$
\begin{array}{cc}
\int_{\Omega_{S}} \boldsymbol{\sigma}(\mathbf{u}): \boldsymbol{\varepsilon}(\delta \mathbf{u}) \mathrm{d} v \Rightarrow \delta \mathbf{U}^{T} \mathbf{K}_{u} \mathbf{U} \quad ; \quad \int_{\Omega_{S}} \rho_{S} \mathbf{u} \cdot \delta \mathbf{u} \mathrm{d} v \Rightarrow \delta \mathbf{U}^{T} \mathbf{M}_{u} \mathbf{U} \\
\frac{1}{\rho_{F}} \int_{\Omega_{F}} \nabla p \cdot \nabla \delta p \mathrm{~d} v \Rightarrow \delta \mathbf{P}^{T} \mathbf{K}_{p} \mathbf{P} & ; \quad \frac{1}{\rho_{F} c_{F}^{2}} \int_{\Omega_{F}} p \delta p \mathrm{~d} v \Rightarrow \delta \mathbf{P}^{T} \mathbf{M}_{p} \mathbf{P} \\
\int_{\Sigma} p \mathbf{n} . \delta \mathbf{u} \mathrm{d} s \Rightarrow \delta \mathbf{U}^{T} \mathbf{C}_{u p} \mathbf{P} & ; \quad \int_{\Sigma} p \delta p \mathrm{~d} s \Rightarrow \delta \mathbf{P}^{T} \mathbf{D}_{p} \mathbf{P}
\end{array}
$$

\subsection{Kelvin-Voigt impedance model}

Due to the frequency complex dependence of the acoustic wall impedance $Z(\omega)$, the previous unsymmetric formulation can be written only in frequency domain and allows to nonlinear system in terms of angular frequency $\omega$. To avoid this problem, a new finite element formulation based on the introduction of an additional scalar unknown, namely the normal fluid displacement field $\eta$ at the dissipative interface, has been recently presented by the authors (Deü et al., 2006; Larbi et al., 2006). In this case, the acoustic wall impedance $Z(\omega)$ is approximated by a Kelvin-Voigt rheological model, i.e. sum of a constant real part and an imaginary part inversely proportional to the frequency: $Z(\omega)=d^{I}+i k^{I} / \omega$ (see Section 2). The local equations of the 
structural-acoustic problem with absorbing material can then be written in terms of structure displacement $\mathbf{u}$, fluid pressure $p$ and normal fluid displacement at the interface $\eta$ :

- Structure

$$
\begin{array}{rlrl}
\operatorname{div} \boldsymbol{\sigma}(\mathbf{u})-\rho_{S} \frac{\partial^{2} \mathbf{u}}{\partial t^{2}} & =\mathbf{0} & & \text { in } \Omega_{S} \\
\boldsymbol{\sigma}(\mathbf{u}) \mathbf{n}^{S} & =\mathbf{F}^{d} & \text { on } \Gamma_{t} \\
\mathbf{u} & =\mathbf{0} & \text { on } \Gamma_{u} \\
\boldsymbol{\sigma}(\mathbf{u}) \mathbf{n}^{S} & =\left[k^{I}(\eta-\mathbf{u} \cdot \mathbf{n})+d^{I}\left(\frac{\partial \eta}{\partial t}-\frac{\partial \mathbf{u}}{\partial t} \cdot \mathbf{n}\right)\right] \mathbf{n} & \text { on } \Sigma
\end{array}
$$

- Fluid

$$
\begin{aligned}
\Delta p-\frac{1}{c_{F}^{2}} \frac{\partial^{2} p}{\partial t^{2}} & =0 & & \text { in } \Omega_{F} \\
\nabla p \cdot \mathbf{n} & =-\rho_{F} \frac{\partial^{2} \eta}{\partial t^{2}} & & \text { on } \Sigma
\end{aligned}
$$

- Interface

$$
p+k^{I}(\mathbf{u} \cdot \mathbf{n}-\eta)+d^{I}\left(\frac{\partial \mathbf{u}}{\partial t} \cdot \mathbf{n}-\frac{\partial \eta}{\partial t}\right)=0 \quad \text { on } \Sigma
$$

The variational formulation of the problem is obtained using the test-function method.

Multiplying Equation [17] by $\delta \mathbf{u} \in C_{u}^{\star}$, applying Green's formula, and finally taking Equations [18] and [20] into account, we have:

$$
\begin{aligned}
& \int_{\Omega_{S}} \boldsymbol{\sigma}(\mathbf{u}): \boldsymbol{\varepsilon}(\delta \mathbf{u}) \mathrm{d} v+k^{I} \int_{\Sigma}(\mathbf{u} \cdot \mathbf{n}) \mathbf{n} \cdot \delta \mathbf{u} \mathrm{d} s+d^{I} \int_{\Sigma}\left(\frac{\partial \mathbf{u}}{\partial t} \cdot \mathbf{n}\right) \mathbf{n} \cdot \delta \mathbf{u} \mathrm{d} s \\
& -k^{I} \int_{\Sigma} \eta \mathbf{n} \cdot \delta \mathbf{u} \mathrm{d} s-d^{I} \int_{\Sigma} \frac{\partial \eta}{\partial t} \mathbf{n} \cdot \delta \mathbf{u} \mathrm{d} s+\int_{\Omega_{S}} \rho_{S} \frac{\partial^{2} \mathbf{u}}{\partial t^{2}} \cdot \delta \mathbf{u} \mathrm{d} v=\int_{\Gamma_{t}} \mathbf{F}^{d} \cdot \delta \mathbf{u} \mathrm{d} s
\end{aligned}
$$

Similarly, multiplying Equation [21] by $\delta p \in C_{p}$, applying Green's formula, and finally taking Equation [22] into account, we obtain:

$$
\frac{1}{\rho_{F}} \int_{\Omega_{F}} \nabla p \cdot \nabla \delta p \mathrm{~d} v+\frac{1}{\rho_{F} c_{F}^{2}} \int_{\Omega_{F}} \frac{\partial^{2} p}{\partial t^{2}} \delta p \mathrm{~d} v+\int_{\Sigma} \frac{\partial^{2} \eta}{\partial t^{2}} \delta p \mathrm{~d} s=0
$$


Finally, we consider the space $C_{\eta}$ of sufficiently regular functions $\eta$ defined in $\Sigma$. Multiplying Equation [23] by $\delta \eta \in C_{\eta}$, we have:

$$
\begin{aligned}
k^{I} \int_{\Sigma} \eta \delta \eta \mathrm{d} s & -k^{I} \int_{\Sigma} \mathbf{u} \cdot \mathbf{n} \delta \eta \mathrm{d} s \\
& +d^{I} \int_{\Sigma} \frac{\partial \eta}{\partial t} \delta \eta \mathrm{d} s-d^{I} \int_{\Sigma} \frac{\partial \mathbf{u}}{\partial t} \cdot \mathbf{n} \delta \eta \mathrm{d} s-\int_{\Sigma} p \delta \eta \mathrm{d} s=0
\end{aligned}
$$

Thus, the variational unsymmetric formulation of the elastoacoustic problem with interface damping consists, for given appropriate initial conditions, in finding $(\mathbf{u}, \eta, p) \in\left(C_{u}^{\star}, C_{\eta}, C_{p}\right)$ such that, $\forall(\delta \mathbf{u}, \delta \eta, \delta p) \in\left(C_{u}^{\star}, C_{\eta}, C_{p}\right)$, Equations [24], [25] and [26] are satisfied.

After discretizing by the finite element method the bilinear forms in Equations [24], [25] and [26, we obtain the following matrix equation of the coupled system:

$$
\begin{aligned}
\left(\begin{array}{ccc}
\mathbf{M}_{u} & \mathbf{0} & \mathbf{0} \\
\mathbf{0} & \mathbf{0} & \mathbf{0} \\
\mathbf{0} & \mathbf{C}_{\eta p}^{T} & \mathbf{M}_{p}
\end{array}\right)\left(\begin{array}{c}
\ddot{\mathbf{U}} \\
\ddot{\mathbf{H}} \\
\ddot{\mathbf{P}}
\end{array}\right)+\left(\begin{array}{ccc}
d^{I} \mathbf{G}_{u} & -d^{I} \mathbf{G}_{u \eta} & \mathbf{0} \\
-d^{I} \mathbf{G}_{u \eta}^{T} & d^{I} \mathbf{G}_{\eta} & \mathbf{0} \\
\mathbf{0} & \mathbf{0} & \mathbf{0}
\end{array}\right)\left(\begin{array}{c}
\dot{\mathbf{U}} \\
\dot{\mathbf{H}} \\
\dot{\mathbf{P}}
\end{array}\right) \\
+\left(\begin{array}{ccc}
\mathbf{K}_{u}+k^{I} \mathbf{G}_{u} & -k^{I} \mathbf{G}_{u \eta} & \mathbf{0} \\
-k^{I} \mathbf{G}_{u \eta}^{T} & k^{I} \mathbf{G}_{\eta} & -\mathbf{C}_{\eta p} \\
\mathbf{0} & \mathbf{0} & \mathbf{K}_{p}
\end{array}\right)\left(\begin{array}{c}
\mathbf{U} \\
\mathbf{H} \\
\mathbf{P}
\end{array}\right)=\left(\begin{array}{c}
\mathbf{F} \\
\mathbf{0} \\
\mathbf{0}
\end{array}\right)
\end{aligned}
$$

where $\mathbf{U}, \mathbf{H}$ and $\mathbf{P}$ are the vectors of nodal values of $\mathbf{u}, \eta$ and $p$ respectively and the new submatrices are given by

$$
\begin{array}{ll}
\int_{\Sigma} p \delta \eta \mathrm{d} s \Rightarrow \delta \mathbf{H}^{T} \mathbf{C}_{\eta p} \mathbf{P} & \quad \int_{\Sigma} \frac{\partial^{2} \eta}{\partial t^{2}} \delta p \mathrm{~d} s \Rightarrow \delta \mathbf{P}^{T} \mathbf{C}_{\eta p}^{T} \ddot{\mathbf{H}} \\
\int_{\Sigma}(\mathbf{u} \cdot \mathbf{n}) \mathbf{n} \cdot \delta \mathbf{u} \mathrm{d} s \Rightarrow \delta \mathbf{U}^{T} \mathbf{G}_{u} \mathbf{U} & ; \quad \int_{\Sigma} \eta \delta \eta \mathrm{d} s \Rightarrow \delta \mathbf{H}^{T} \mathbf{G}_{\eta} \mathbf{H} \\
\int_{\Sigma} \eta \mathbf{n} \cdot \delta \mathbf{u} \mathrm{d} s \Rightarrow \delta \mathbf{U}^{T} \mathbf{G}_{u \eta} \mathbf{H} & ; \quad \int_{\Sigma} \mathbf{u} \cdot \mathbf{n} \delta \eta \mathrm{d} s \Rightarrow \delta \mathbf{H}^{T} \mathbf{G}_{u \eta}^{T} \mathbf{U}
\end{array}
$$

This formulation has the advantage that it can be solved in frequency and in time domain by introducing only one unknown per node on the damping interface (normal fluid displacement $\eta$ ). On the other hand, it has the disadvantage of yielding unsymmetric matrices. The symmetric formulation can be obtained through the introduction of an intermediate unknown field, namely fluid displacement potential $\varphi$ defined up to an additive constant (Morand et al., 1995; Deü et al., 2006). 


\section{Example: dissipative acoustic pipe submitted to a pressure load}

In this example, a pressure load is applied to the left edge of a straight pipe (of length $A=5 \mathrm{~m}$ and width $B=0.5 \mathrm{~m}$ ) containing air (density $\rho_{F}=1 \mathrm{~kg} / \mathrm{m}^{3}$, speed of sound $c_{F}=340 \mathrm{~m} / \mathrm{s}$ ) and with an absorption wall at the right edge (Figure 4). This absorbing boundary is modeled by the previously described wall acoustic impedance $Z(\omega)=d^{I}+i k^{I} / \omega$. The damping parameters, given in Section 2.2, are chosen in order to attenuate the vibration amplitudes without shifting the frequencies in a too significant way. The considered harmonic excitation has the following form $p^{d}(t)=$ $p_{0} \sin \left(2 \pi t / T_{1}\right)$ with $1 / T_{1}=200 \mathrm{~Hz}$ and $p_{0}=1000 \mathrm{~Pa}$. Moreover, the transient response of the acoustic pipe is computed using a direct time integration method.

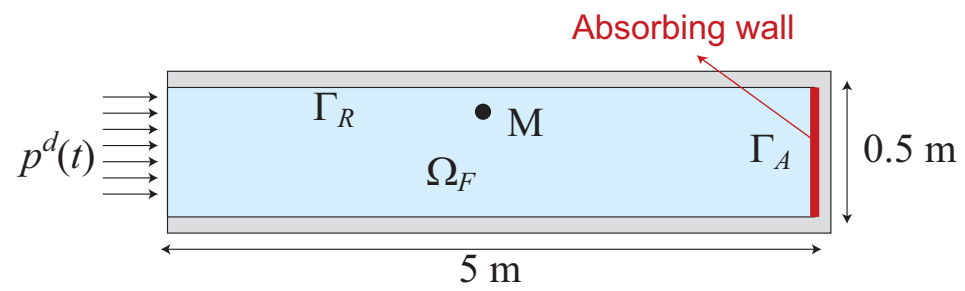

Figure 4. Straight pipe submitted to a pressure load at the left end

Figure 4 shows the frequency responses of the damped and undamped acoustic pipe at the point $\mathrm{M}(2.5 \mathrm{~m}, 0.4 \mathrm{~m})$. This figure indicates that the damping significantly reduced the first resonant peaks, with the largest reductions achieved after the excitation (at $200 \mathrm{~Hz}$ ). Moreover, it can be observed that the absorbing boundary caused a small shift of the resonant frequencies. This is due to the spring effect of the KelvinVoigt model used for the impedance.

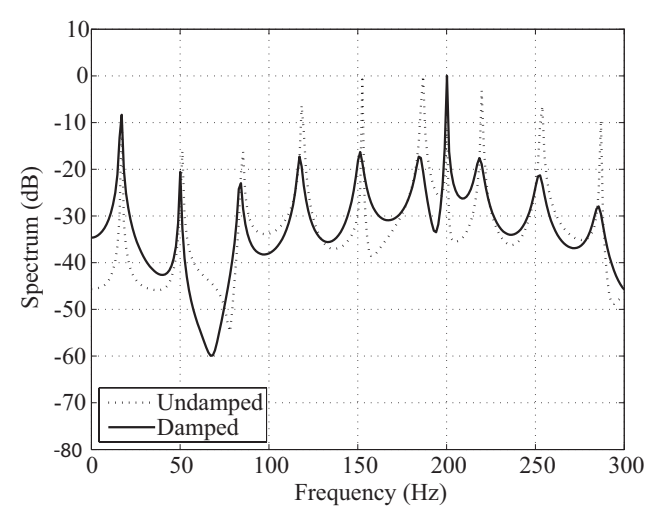

Figure 5. Pipe frequency responses to harmonic pressure in the damped and undamped cases 


\section{Conclusions}

Finite element formulations of interior vibroacoustic coupled problems with absorbing interfaces have been presented in this work. The dissipative interface is modeled by wall acoustic impedance in a limited frequency range. The used technique is based on the prediction of the surface impedance parameters from a Biot-Allard poroelastic model. This approach, combined with an original structural-acoustic finite element formulation developed by the authors, is tested on an acoustic pipe example with damping interface.

\section{References}

Allard J.-F., Propagation of Sound in Porous Media: Modelling Sound Absorbing Materials, Elsevier Applied Science, London, 1993.

Atalla N., Panneton R., Debergue P., “ A mixed displacement-pressure formulation for poroelastic materials", Journal of the Acoustic Society of America, vol. 104, n 3, p. 1444-1452, 1998.

Bermúdez A., Rodríguez R., “ Modelling and numerical solution of elastoacoustic vibrations with interface damping”, International Journal for Numerical Methods in Engineering, vol. 46, n 10, p. 1763-1779, 1999.

Davidsson P., Structure-acoustic analysis; Finite element modelling and reduction methods, Phd thesis, Lund University, 2004.

Davidsson P., Sandberg G., “ A reduction method for structure-acoustic and poroelastic-acoustic problems using interface-dependent Lanczos vectors", Computer Methods in Applied Mechanics and Engineering, vol. 195, n 17-18, p. 1933-1945, 2006.

Deü J.-F., Larbi W., Ohayon R., “ Dissipative interface modeling for vibroacoustic problems - A new symmetric formulation”, in C. M. Soares, J. Martins, H. Rodrigues, J. Ambrósio (eds), Computational Mechanics - Solids, Structures and Coupled Problems, Springer, Dordrecht, Netherlands, p. 413-428, 2006.

Göransson P., “ A 3-D, symmetric, finite element formulation of the Biot equations with application to acoustic wave propagation through an elastic porous medium", International Journal for Numerical Methods in Engineering, vol. 41, $\mathrm{n}^{\circ}$ 1, p. 167-192, 1998.

Kehr-Candille V., Ohayon R., “ Elastoacoustic damped vibrations - finite element and modal reduction methods", in O. Z. P. Ladevèze (ed.), New Advances in Computational Structural Mechanics, Elsevier, Amsterdam, Netherlands, p. 321-334, 1992.

Larbi W., Deü J.-F., Ohayon R., “ A new finite element formulation for internal acoustic problems with dissipative walls", International Journal for Numerical Methods in Engineering, vol. 68, n³, p. 381-399, 2006.

Morand H.-P., Ohayon R., Fluid-Structure Interaction, Wiley, New York, 1995.

Pierce A., Acoustics: An Introduction to Its Physical Principles and Applications, Acoustical Society of America, New York, 1989. 